\title{
Notfälle im Rettungsdienst und in der Klinik: Gefäßzugänge bei Kindern
}

Tomohiro Yamamoto, Ehrenfried Schindler

\author{
Der i.v. Gefäßzugang muss v. a. im Notfall adäquat beherrscht werden. Gerade aber \\ bei kleinen Kindern oder Säuglingen ist die Punktion peripherer Venen mitunter \\ schwierig und in einer zeitkritischen Situation häufig auch sehr stressbelastet. Hier \\ muss der Anwender Alternativen kennen und Hilfsmittel beherrschen können. Dieser \\ Beitrag geht auf die verschiedenen Gefäßzugänge ein, diskutiert Alternativen und \\ beschreibt gängige Techniken.
}

\section{Einleitung}

Der i. v. Gefäßzugang wird gelegt, um Medikamente und/ oder Flüssigkeiten zuführen zu können. Bei den meisten erwachsenen Patienten kann man periphere Venen i.d.R. visuell identifizieren oder manuell tasten, besonders wenn sie sich mit der Anwendung eines Stauschlauchs adäquat füllen. Bei Neugeborenen, Säuglingen und kleinen Kindern ist dies oftmals nicht so einfach: Die sehr kleinen Gefäße sind häufig nicht gut zu sehen und zu tasten, auch weil das Oberhautfettgewebe diese überdeckt. Noch schwieriger wird es besonders in Notfallsituationen bei kritisch kranken Patienten mit reduziertem Kreislauf. Präklinische Notfallsituationen mit pädiatrischen Patienten können alle Teilnehmer des Rettungsdienstes treffen, auch wenn sie nicht speziell für Kindernotfälle ausgebildet sind. In diesem Zusammenhang müssen etablierte Techniken beherrscht werden, aber auch alternative Gefäßzugangsmöglichkeiten bekannt sein.

In außerklinischen Notfallsituationen kann die rasche Etablierung eines Gefäßzugangs lebensrettend sein. Nach aktuellen Empfehlungen werden in solchen Situationen wenn möglich die peripheren Verweilkanülen oder alternativ dazu die Anlage eines intraossären (i.o.) Gefäßzugangs empfohlen [1]. Berichte bestätigen, dass i. o. Zugänge im Kreislaufstillstand oder unter Reanimation erfolgreicher und schneller als i. v. Zugänge etabliert werden können [2-8]. Obwohl zahlreiche transportable UItraschallgeräte entwickelt wurden, die auch im Rettungsdienst eingesetzt werden, sind diese zurzeit bei außerklinischen Notfallsituationen noch nicht flächendeckend verfügbar. Nach Stabilisierung der Vitalfunktionen und Transport ins Krankenhaus kann die Anlage eines periphervenösen (PVK) oder zentralvenösen Katheters (ZVK) erwogen werden, um einen bereits liegenden i. o. Zugang zu ersetzen.

\begin{abstract}
Merke
In Notfallsituationen wird die periphere Verweilkanüle oder der i. o. Gefäßzugang empfohlen.
\end{abstract}

Die Anwendung des Ultraschalls zu Gefäßpunktion nimmt einen immer größeren Stellenwert ein. Daher werden im Folgenden auch ultraschallbasierte Techniken vorgestellt. Die Sonografie kann die Anlage von peripheren Verweilkanülen und/oder zentralvenösen Kathetern beim pädiatrischen Patienten in innerklinischen Notfallsituationen oder bei schwierigen Venenverhältnissen erheblich erleichtern.

\section{Intraossäre Gefäßzugänge}

Die aktuelle Leitlinie des European Resuscitation Council (ERC) empfiehlt den i.o. Gefäßzugang als alternativen Zugangsweg zum Gefäßsystem unter Reanimation bei pädiatrischen Patienten [1]. In dieser Leitlinie wird gefordert, dass nach max. 2 Versuchen (oder 2 min), einen peripheren i.v. Zugang zu legen, alternativ der i. o. Zugang erwogen werden muss ( $\triangleright$ https://www.resus.org.uk/ resuscitation-guidelines/prehospital-resuscitation/).

\section{Vorgehen}

Das Knochenmark steht durch reichhaltige Kapillarisierung in direkter Verbindungen zum Gefäßsystem. Es bietet sich an, im Notfall einen Gefäßzugang zu etablieren und direkt vom Knochenmark das 1. Blutpräparat während der i. o. Punktion im Kreislaufstillstand oder unter Reanimation zur Laborbestimmung abzunehmen [9, 10]. Die ausgeprägte Vaskularisierung des Knochenmarks ist ein Grund für die ausgedehnten Blutungen nach Frakturen großer Knochen ( $\bullet$ Tab. 1). Durch die i. o. Zugänge macht man sich diese Vaskularisierung zunutze. Über diese können Kathecholamine, Infusionen und Blutprodukte sicher verabreicht werden $[11,12]$. Intraossär applizierte 


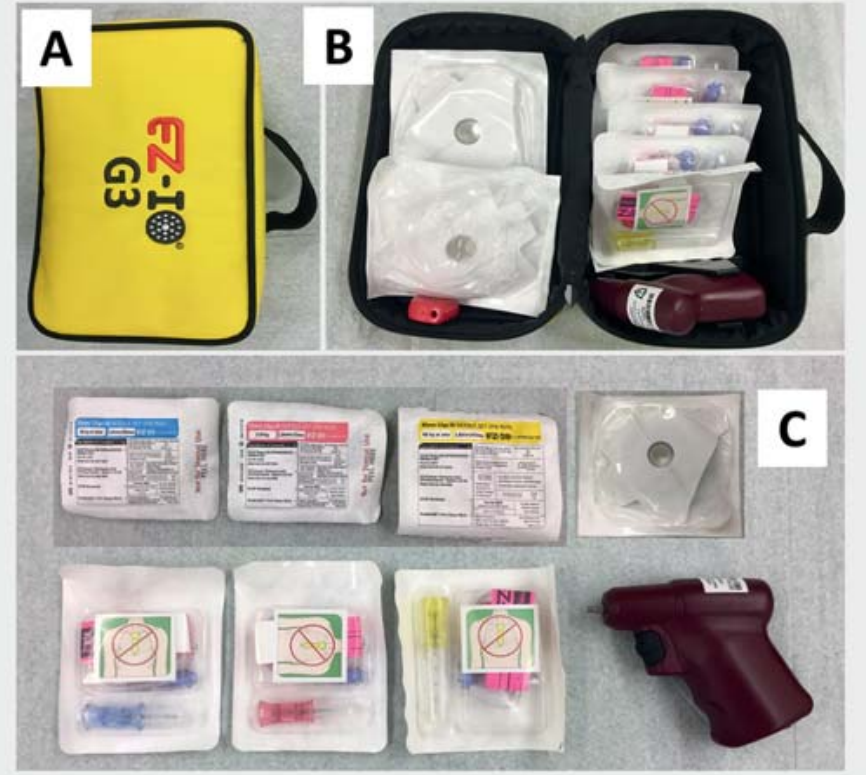

- Abb. 1 Beispiel eines semiautomatischen Systems für die Anlage von i. o. Zugängen (EZ-IO ${ }^{\circledR}$, Teleflex Medical GmbH). (a) Aussehen der Tasche. (b, c) Inhalt der Tasche: Akkubohrer, i. o. Nadeln in verschiedenen Größen und i. o. Nadel-Stabilizer (Pflaster).

Medikamenten erreichen zügig den zentralen Kreislauf. Die Blutkonzentration so verabreichter Pharmaka ist vergleichbar mit i.v. gegebener Medikation [13]. Bei wachen Patienten sollte man bedenken, dass die Injektion ins Knochenmark sehr schmerzhaft ist, daher wird empfohlen, vorab ein Lokalanästhetikum zu verabreichen.

\section{Merke}

Beim i.o. Gefäßzugang macht man sich die starke Vaskularisierung des Knochenmarks zunutze. Dadurch können Flüssigkeiten sicher verabreicht werden und erreichen zügig den zentralen Kreislauf.

\section{Geringe Komplikationsraten}

Das Material für i. o. Zugänge sollte in jedem OP oder in außerklinischen Rettungssystemen vorhanden sein. Der große Vorteil ist, dass sich i.o. Zugänge bei geringen Komplikationsraten leicht erlernen lassen [2-8]. Es wird empfohlen, in klinikinternen Handlungsempfehlungen festzuhalten, wie lange der Versuch dauern soll, einen konventionellen Gefäßzugang beim Kind zu legen [14]. Bei Kreislaufstillstand sollte sofort ein i. o. Zugang gelegt werden. Auch bei in Notfallsituationen gelegten i. o. Zugängen sind die Komplikationsraten bei pädiatrischen Patienten gering [2-8].
- Tab. 1 Möglicher Blutverlust nach Frakturen bei erwachsenen Patienten.

\begin{tabular}{|l|c|}
\hline Blutungsort & Blutungsmenge \\
\hline Humerus & $300 \mathrm{ml}$ \\
\hline Tibia & $500 \mathrm{ml}$ \\
\hline Femur & $1000 \mathrm{ml}$ \\
\hline Becken & $2000 \mathrm{ml}$ \\
\hline
\end{tabular}

\section{Verschiedene Systeme}

Während im pädiatrischen Bereich lange Jahre nur manuelle Nadelsysteme verfügbar waren, sind nun auch verschiedene semiautomatische Systeme auf dem Markt. Unzweifelhaft sind die semiautomatischen i.o. Systeme in der außerklinischen Notfallsituation wesentlich schneller und effektiver als die manuell einzubohrenden Nadeln und zeigen weniger Komplikationen [15]. > Abb. 1 zeigt ein Beispiel eines semiautomatischen Systems, das in vielen Abteilungen eingesetzt wird [16]. Bei einem weiteren semiautomatischen System schnellt eine Nadel mithilfe eines Federwerks automatisch durch Haut und Knochen, wenn das System über dem Zielknochen ausgelöst wird (Bone Injection Gun = B.I.G). Die Autoren bevorzugen die semiautomatische Methode mittels Bohrer oder alternativ dazu besonders bei kleinsten Säuglingen < $3 \mathrm{~kg} / \mathrm{KG}$ die manuelle Methode mittels einer speziellen i. o. Kanüle. Der Vorteil liegt in einer gefühlvolleren Punktion des weichen Knochens mit einer Art „loss of resistance“Technik.

\section{Merke \\ Zugangswege für die i. o. Nadelanlage sind z. B. der distale Femur, die proximale und distale Tibia, die Spina iliaca anterior superior und der proximale Humerus ( Abb. 2).}

\section{Punktionsorte}

- Tab. 2 listet die charakteristischen Merkmale der möglichen Punktionsorte auf. Die erste Wahl stellt die proximale Tibia dar [1-8], da die flache Seite des Knochens einfach zu tasten ist und die Nadel stabil fixiert werden kann. Die Einstichstelle ist 1-2 cm medial und distal der Tuberositas tibiae ( $\bullet$ Abb. $\mathbf{2}$ b). Der i. o. Zugang sollte nur 1-mal pro Knochen versucht werden.

\section{Kontraindikationen}

Als Kontraindikationen für i. o. Zugänge gelten Infektionen nahe der Einstichstelle und der Verdacht auf eine Fraktur des zu punktierenden Knochens ( $\bullet$ Infobox: Kontraindikation für die i.o. Zugänge). Die Punktion der Wachstumsfuge bei Kindern ist unbedingt zu vermeiden. 
- Tab. 2 Punktionsorte für die intraossäre Anlage eines Gefäßzugangs bei Kindern.

\begin{tabular}{|l|l|}
\hline Einstichstelle & Anmerkungen \\
\hline Femur distal & $\begin{array}{l}\text { - } \\
\text { eher problematisch wegen Muskula- } \\
\text { tur und des subkutanen Fettgewebes } \\
\text { - schlechter tastbar }\end{array}$ \\
\hline Tibia proximal & $\begin{array}{l}\text { - erste Wahl } \\
\text { - flache Stelle } 1-2 \mathrm{~cm} \text { medial und distal } \\
\text { der Tuberositas tibiae }\end{array}$ \\
\hline Tibia distal & $\begin{array}{l}\text { flache Stelle } 1-2 \mathrm{~cm} \text { (1 Fingerbreite) } \\
\text { proximal vom inneren Knöchel }\end{array}$ \\
\hline $\begin{array}{l}\text { Spina iliaca an- } \\
\text { terior superior }\end{array}$ & $\begin{array}{l}\text { - } 1 \text { cm proximal der Tuberositas } \\
\text { Humerus } \\
\text { proximal }\end{array}$ \\
\hline
\end{tabular}

\section{INFO}

Kontraindikationen für die i. o. Zugänge

- Infektionen oder starke Verschmutzung der Einstichstelle

- mehrfache, frustrane Punktionsversuche

- Fraktur oder Verdacht auf eine solche

\section{Fallberichte}

Es wurden Fälle berichtet, bei denen pädiatrischen Patienten mit Kompartmentsyndrom und Osteomyolitis nach Anlage eines i. o. Zugangs der Unterschenkel amputiert werden musste $[8,17,18]$. Nach einem weiteren Fallbericht wurde bei einem Säugling, trotz ordnungsgemäßer Anwendung nach Herstellerangabe und korrekter Technik, die Hinterkante der Tibia perforiert, wodurch Medikamente und Flüssigkeit subkutan infundierten. In diesem Fall war die Nadel zu lang für den Patienten [17].

- Abb. 3 zeigt einen Fall nach Anlage eines i. o. Zugangs in die proximale Tibia unter Reanimation bei einem Säugling mit angeborenem Herzfehler. Im Bereich der Einstichstelle entwickelte sich eine schwere subkutane Nekrose mit nachfolgendem Kompartmentsyndrom im Unterschenkel. Der Patient war zu diesem Zeitpunkt 30 Tage alt und zeigte eine stark reduzierte linksventrikuläre Funktion bei hypertropher Kardiomyopathie mit multiplen Ventrikelseptumdefekten und einem persistierenden Foramen ovale. Ein erfahrener Facharzt führte die i. o. Punktion unter Herz-Druck-Massage durch und bestätigte die Lage der Kanüle nach gängigen Kriterien. Über diesen Zugang wurden Ringerlösung und Medikamente inklusive Adrenalin infundiert. Nach Stabilisierung der Vitalfunktionen und Transport auf die kardiochirurgische Intensivstation legten die Ärzte einen zentralen Venenkatheter und entfernten die i. o. Nadel.

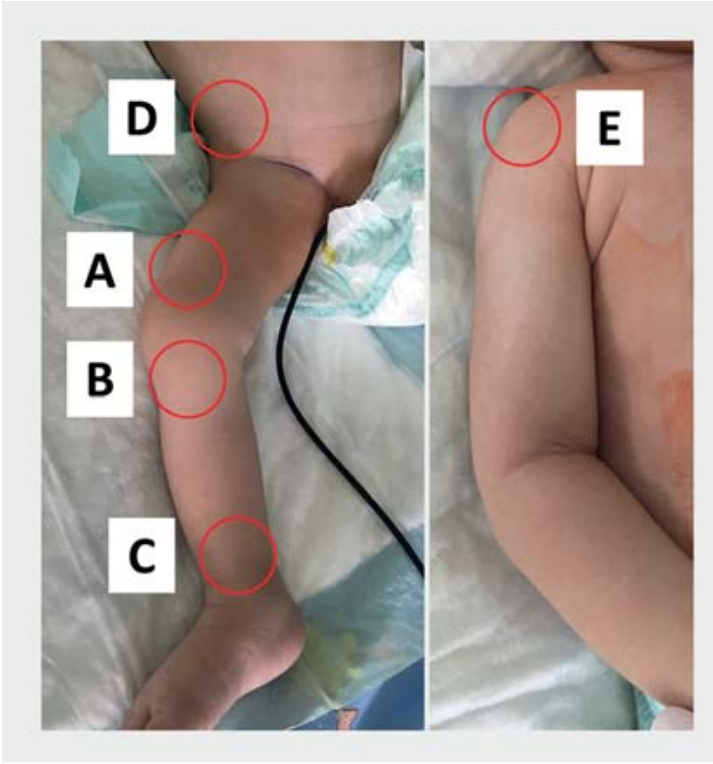

Abb. 2 Punktionsorte für den i. o. Zugang.

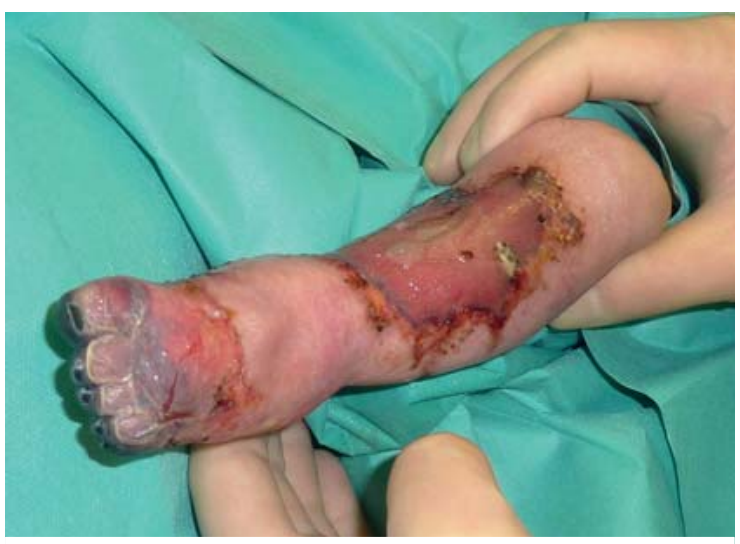

- Abb. 3 Beispiel einer schweren subkutanen Nekrose und Kompartment Syndrom nach Anlage eines i. o. Zuganges in die proximale Tibia unter Reanimationsbedingungen mit nachfolgender ECMO Anlage.

Ein weiterer lesenswerter Fallbericht von Suominen et al. [17] weist zu Recht darauf hin, dass die zur Verfügung stehenden i. o. Nadeln der entsprechenden semiautomatischen Systeme nach Altersgruppen ausgewählt werden sollen. Das bedeutet, dass Kinder am oberen oder unteren Rand dieser Altersgruppen mit besonderer Sorgfalt behandelt werden müssen, da die Knochendichte und -dicke deutlich je nach Alter variiert. Die Autoren haben den durchschnittlichen Durchmesser des medullären Raums der proximalen Tibia in verschiedenen Altersgruppen anhand von Röntgenaufnahmen vermessen und kamen zu folgenden Ergebnissen ( Tab. 3) [17]: 
- Tab. 3 Durchmesser des medullären Raums der proximalen Tibia in verschiedenen Altersgruppen. Nach [17].

\begin{tabular}{|l|l|}
\hline Altersgruppe & $\begin{array}{l}\text { Durchmesser des medullären } \\
\text { Raums der proximalen Tibia }\end{array}$ \\
\hline Neonaten & $7 \mathrm{~mm}$ \\
\hline 1 -12 Monate alte Kinder & $10 \mathrm{~mm}$ \\
\hline 3-4 Jahre alte Kinder & $12 \mathrm{~mm}$ \\
\hline
\end{tabular}

- $7 \mathrm{~mm}$ bei Neonaten

- $10 \mathrm{~mm}$ bei 1-12 Monate alten Kindern

- 12 mm bei 3-4 Jahre alten Kindern

Dieser Fallbericht empfiehlt, dass die richtige Position der i.o. Nadeln mittels Aspiration von Knochenmark und Bolusgabe kristalloider Lösung bestätigt werden soll. Die Umgebung des Punktionsorts muss sorgfältig auf Zeichen der subkutanen Injektion (Schwellung, Rötung) inspiziert werden. Bei der Indikation zur therapeutischen Hypothermie sollte der i.o. Zugang wegen des möglichen Problems der peripheren Vasokonstriktion mit Möglichkeit einer peripheren Minderperfusion rasch entfernt werden.

\section{Merke}

Für die außerklinische Notfallsituation wird empfohlen, den i. o. Zugang nach Stabilisierung der Vitalfunktionen und Transport ins Krankenhaus durch einen entsprechenden peripheren oder zentralen Venenkatheter zu ersetzen [17].

\section{Limitationen der Landmarkentechnik}

Traditionell werden zentralvenöse Zugänge mithilfe anatomischer Landmarken gelegt. Im Fall der häufig praktizierten Punktion der V. jugularis interna versucht man, die Halsmuskulatur zu identifizieren und anhand der Pulsation der A. carotis interna die ungefähre Lage der V. jugularis interna zu bestimmen. Es ist jedoch beschrieben, dass $V$. juguralis interna und $A$. carotis communis bei pädiatrischen Patienten in ca. $10 \%$ der Fälle nicht nebeneinander sondern hintereinander liegen [26]. Ähnliches wird über die Lagebeziehung bei $\mathrm{V}$. femoralis und A. femoralis berichtet [27]. Die Identifikation zentraler Venen kann weiter erschwert werden, wenn die Venen durch Thrombose oder Hämatome komprimiert oder verlagert sind, was durch die Landmarkentechnik oft nicht leicht ersichtlich ist. Bei Säuglingen oder kleinen pädiatrischen Patienten kommt erschwerend hinzu, dass die Dimensionen um ein vielfaches kleiner sind im Vergleich zu Erwachsenen. Das erklärt, warum die Erfolgsrate z.B. bei V.-juguralis-Punktion mit Landmarkenmethode nur bei 70-90\% liegt [28-31]. Es ist auch berichtet, dass bei pädiatrischen Patienten im Notfall in > 30\% der
Versuche mithilfe der Landmarkenmethode die V. femoralis mit der $A$. femoralis verwechselt und falsch punktiert wurde [32].

\section{Merke \\ Die Landmarkentechnik ist oftmals nicht eindeutig und sollte daher mithilfe der Sonografie validiert werden.}

\section{Katheteranlagen mit Sonografie}

Die Verwendung von Ultraschall zur Identifikation von Blutgefäßen und während der Punktion ist weit verbreitet und in vielen Zentren zu Recht zum Standard für zentralvenöse Katheteranlagen erhoben worden. Die Ultraschalltechnik kann jedoch nicht nur bei der Punktion zentraler Gefäße hilfreich sein, sondern auch bei der Anlage von peripheren Venenkathetern (PVK). Außerdem werden alternative „Beleuchtungssysteme“ kommerziell angeboten, die mit Licht unterschiedlicher Wellenlängen und Farben helfen sollen, oberflächliche Venen zu identifizieren.

\section{Vergleich mit LED und Nahinfrarotlicht}

Berichten zufolge verbessert sich die Erfolgsrate der Anlage von PVK beim 1. Versuch durch die Nutzung eines LED-Transilluminators (z.B Veinlite ${ }^{\circledR}$ [Translite LLC], Wee Sight $^{\mathrm{TM}}$ [Phillips]; $>$ Abb. 4) bzw. die Punktionsgeschwindigkeit wird erhöht $[19,20]$. Nach unserer Erfahrung scheitert diese Methode jedoch häufig, gerade bei schwierigen Venenverhältnissen bei Säuglingen oder kleinen pädiatrischen Patienten mit prominentem subkutanem Fett oder nach Hämatom durch frustrane Punktionsversuche. Es wurde ebenso berichtet, dass die Anwendung von Nahinfrarotlicht für die PVK-Anlage hilfreich sein kann. Besonders aber bei schwieriger Punktion sind die Erfolgsraten dieser Methode nicht überzeugend [21]. Deutlich erfolgversprechender scheint die direkte Identifikation und Visualisierung auch tieferer peripherer Venen mittels Sonografie zu sein [22,23]. Eine Studie über pädiatrische Patienten in Notfallambulanzen berichtete, dass der Versuch bei den schwierigen peripheren Venen durchschnittlich um 8 min verkürzt ist und die Erfolgsrate mit dem 1. Versuch verbessert wird [24].

\section{Anwendungen der Sonografie}

Für die Ultraschalltechnik dienen in 1. Linie die V. saphena magna oder die subkutanen Venen in der Ellenbeuge als Zielgefäße, da ( $>$ Abb. 5) [25]

- diese Venen tiefer im Fettgewebe liegen,

- bei der Punktion relativ gut fixiert werden können und

- mittels Ultraschall darstellbar sind.

Häufig werden diese dünnen Venen durch die Punktionsnadel selbst komprimiert, sodass ein Durchstechen des Gefäßes unter Sicht mit anschließendem Rückzug häufig 


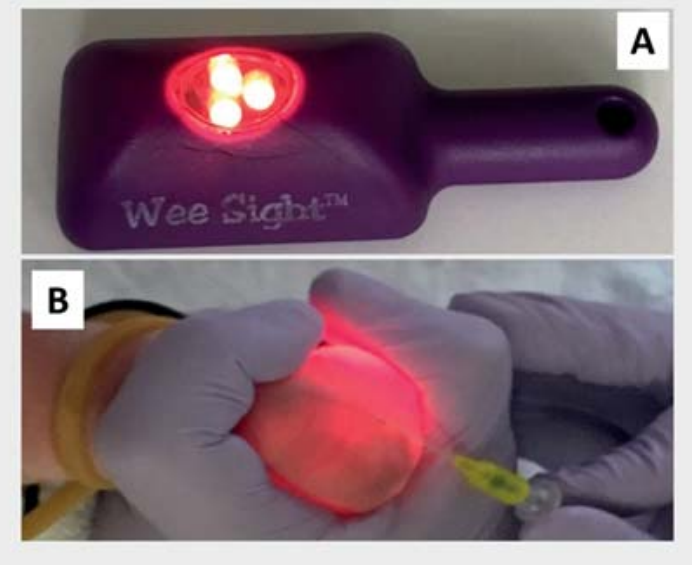

- Abb. 4 Transillumination: Die „Durchleuchtung“ von der Unterseite der Hand aus kann kleinste Venen und Arterien sichtbar machen, sofern kein Hämatom von Vorpunktionsversuchen vorhanden ist.

erfolgversprechender ist. Nach unserer Erfahrung kann die Ultraschalltechnik auch an anderen Körperstellen wie dem Unterarm angewendet werden. Die Ultraschallfrequenz sollte so hoch wie möglich gewählt werden. Wir empfehlen > $10 \mathrm{MHz}$, da die Zielgefäße sehr flach liegen ( $\triangleright$ Abb. 5) und eine höhere Ultraschallfrequenz die Identifikation dieser Strukturen erleichtert.

Während bei zentralisierten oder instabilen pädiatrischen Patienten mitunter viel Zeit vergehen kann, bis ein peripherer Gefäßzugang gelegt ist, kann die Punktion zentraler Venen vom Geübten mittels Ultraschall wesentlich schneller erfolgen. Auch zum Wechsel einer zuvor gelegten i. o. Nadel ist diese Anwendung sinnvoll.

\section{Merke}

Die Ultraschalltechnik ist sowohl bei der Anlage zentraler als auch peripherer Venenkatheter hilfreich und vor allem bei pädiatrischen Patienten klinischer Standard.

\section{Vorteile der Sonografie}

Die Versuche, einen Gefäßzugang beim Kind zu schaffen, sollen so wenig invasiv und schnell wie möglich sein. Für die zügige und sichere Punktion bietet sich gerade hier die Anwendung des Ultraschalls als Hilfsmittel an und ist bereits im innerklinischen Alltag nicht mehr zu ersetzen [33]. Der größte Verteil bei der Anwendung von Sonografie ist die direkte nicht invasive Visualisierung der Zielgefäße. Lokalisation, Größe und Füllungszustand können ebenso beurteilt werden wie die zu erwartende Punktionstiefe.
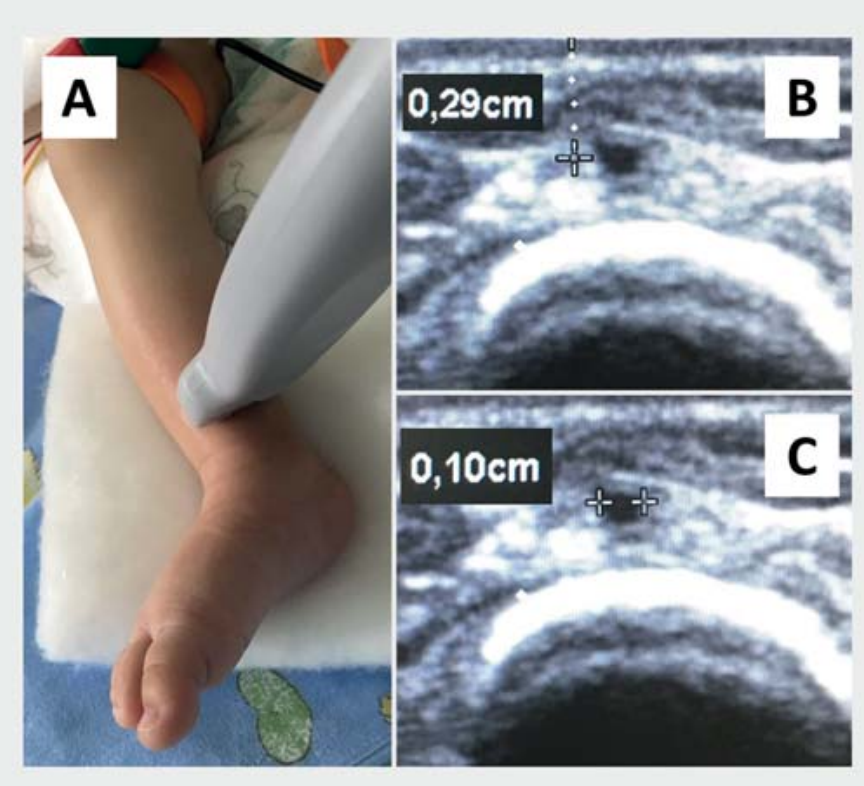

- Abb. 5 Darstellung der V. saphena magna mittels Ultraschall bei einem Neugeborenen (3,2 kg KG). (a) Schallkopf auf V. saphena magna. (b) V. saphena magna in $3 \mathrm{~mm}$ Tiefe. (c) Vermessung der V. saphena magna (1 mm). Beachte: Der Außendurchmesser einer bei Säuglingen üblichen Venenverweilkanüle ist $24 \mathrm{G}=0,7 \mathrm{~mm}$ bzw. $22 \mathrm{G}=0,9 \mathrm{~mm}$.

\section{Methoden}

Derzeit werden 2 Methoden beschrieben:

- "Prescan-Ultrasound-guided-Methode"

- "real-time-Ultrasound-guided-Technik"

Bei der „Prescan-Ultrasound-guided-Methode“ wird das Zielgefäß vor der Punktion einmal identifiziert und möglicherweise markiert. Die Punktion erfolgt dann ohne aufgesetzten Ultraschallkopf. Im Unterschied dazu kann man mit der „real-time-Ultrasound-guided-Technik“ die Punktionskanüle und das Zielgefäß im kompletten Verlauf immer sehen. Es wird berichtet, dass mit der „real-timeUltrasound-guided-Technik“ die Dauer der Punktion deutlich kürzer ist und weniger Versuche nötig sind als mit der „Prescan-Ultrasound-guided-Methode“ [34]. Ein linearer, möglichst kleiner Schallkopf mit hoher Frequenz $(8-13 \mathrm{MHz})$ ist adäquat bei pädiatrischen Patienten, da auch die großen, zentralen Venen relativ oberflächlich verlaufen und durch hochfrequente Schallköpfe besser zu visualisieren sind.

\section{Erfolgsraten}

Die V. jugularis ist auch bei pädiatrischen Patienten der gebräuchlichste Punktionsort. Es wird in einigen Publikationen betont [28-31], dass die Erfolgsrate mit der „realtime-Ultrasound-guided-Technik“ deutlich verbessert wird (ca. 80\% Erfolg mit Landmarkenmethode, nahezu $100 \%$ mit „real-time-Ultrasound-guided-Technik“), wenn die Anwender zuvor wenig Erfahrungen mit der tradi- 


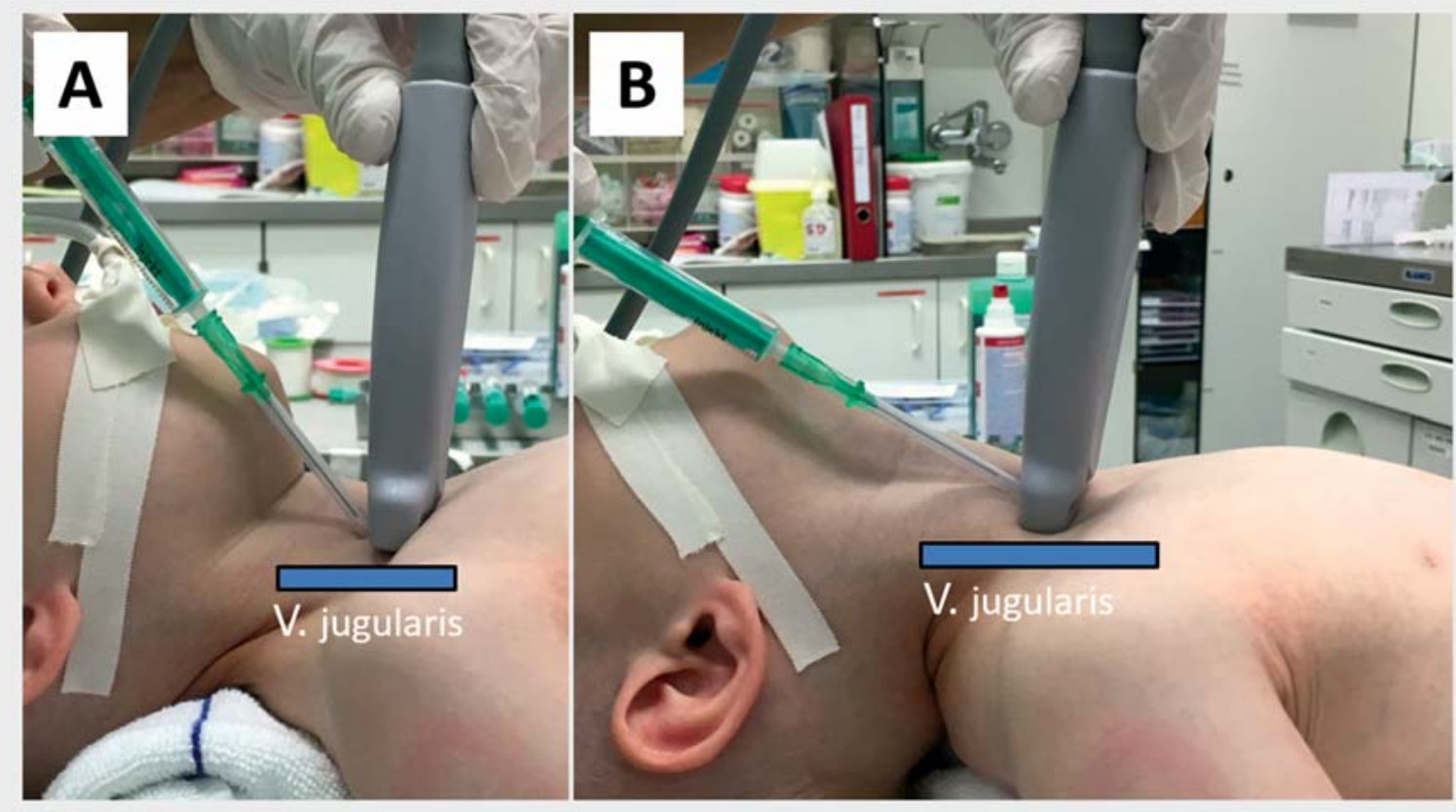

- Abb. 6 Optimale Kopflagerung des Patienten bei der zentralvenösen Punktion mittels Ultraschall. (a) Falsche Lagerung des Kopfes. Die fehlende Überstreckung verhindert einen optimalen Einstichwinkel. (b) Der Kopf des Patienten ist leicht überstreckt, sodass der Punktionswinkel flach (ca. $45^{\circ}$ ) erfolgen kann.

tionellen Landmarkenmethode hatten [28,29]. Einige Autoren konnten zeigen, dass sich die Erfolgsrate mit der „real-time-Ultrasound-guided-Technik“ verschlechtert (ca. 90\% Erfolgsrate mit Landmarkenmethode, ca. 75\% mit „real-time-Ultrasound-guided-Technik“), wenn die Anästhesisten zuvor langjährig Erfahrungen mit der Landmarkenmethode erworben hatten $[30,31]$. Diese Autoren vermuteten, dass Vorerfahrung mit der traditionellen Methode hinderlich beim Erlernen der neuen ultraschallgestützten Methode sei. In jedem Fall fordern sie entsprechende theoretische und praktische Übungen.

\section{Vorgehen}

Die Verwendung der ultraschallgestützten Technik wird schon deshalb dringend empfohlen, da die anatomische Lagebeziehung von V. jugularis interna und A. carotis communis nicht unerheblich variiert $[35,36]$ und sich durch die Kopfposition der Patienten verändern kann [37]. Für den Erfolg ist die Lagerung des Patienten sehr wichtig. Eine Unterlage (z.B eine Rolle oder ein Handtuch) werden unter die Schulter des Patienten gelegt, um den Kopf des Patienten nach hinten beugen zu können. Durch diese Lagerung wird der Hals des Patienten leicht gestreckt und exponiert die Halsgefäße entsprechend. Zudem ermöglicht diese Lagerung einen flacheren Einstichwinkel, was die Erfolgsrate weiter verbessern kann ( $\triangleright$ Abb. 6a). Ohne leichte Überstreckung des Kopfes

- ist das Kinn des Patienten im Weg und

- wird der Einstichwinkel der Punktionskanüle zu steil.
Dadurch wird das nachfolgende Einführen des SeldingerDrahts erschwert oder unmöglich gemacht ( $\mathbf{A} \mathbf{A b} \mathbf{b} \mathbf{6} \mathbf{b}$ ). Die Kopfrotation nach links (bei rechtsseitiger Punktion) kann zudem den relativen Durchmesser der V. jugularis interna vergrößern und die überlappend liegende V. jugularis und A. carotis communis kommen eher nebeneinander zum liegen [38]. Um den Durchmesser der V. jugularis interna weiter zu vergrößern, kann man eine Hilfsperson bitten, Druck auf die Leber auszuüben, da diese beim Säugling ein großes Blutreservoir ist. Kopftieflage, die beim erwachsenen Patienten hilfreich sein kann, bringt beim Kind keine Verbesserung und kann unterbleiben.

\section{Kritik}

Ein häufig geäußerter Kritikpunkt an der ultraschallgesteuerten Punktion ist, dass man damit die „traditionelle“, an anatomischen Landmarken orientierte Technik verlernen könnte. Die Anwendung eines bildgebenden Verfahrens bietet nach Meinung der Autoren jedoch die einmalige Chance, die Lagebeziehung von Nerven und Gefäßen idealerweise mit den zuvor identifizierten anatomischen Landmarken abzugleichen. Ein so strukturiertes Ausbildungsprogramm bietet die Chance, dass sich der Lernende zuvor an anatomischen Landmarken orientiert und die Anatomie der Gefäße oder Nerven imaginiert, bevor man den Schallkopf auf den Patienten legt und anschließend seine Imagination und das Echobild vergleicht. Dadurch kann man sowohl die Landmarkmethode als auch die ultraschallgestützte Technik gleichzeitig erlernen und trainieren. 


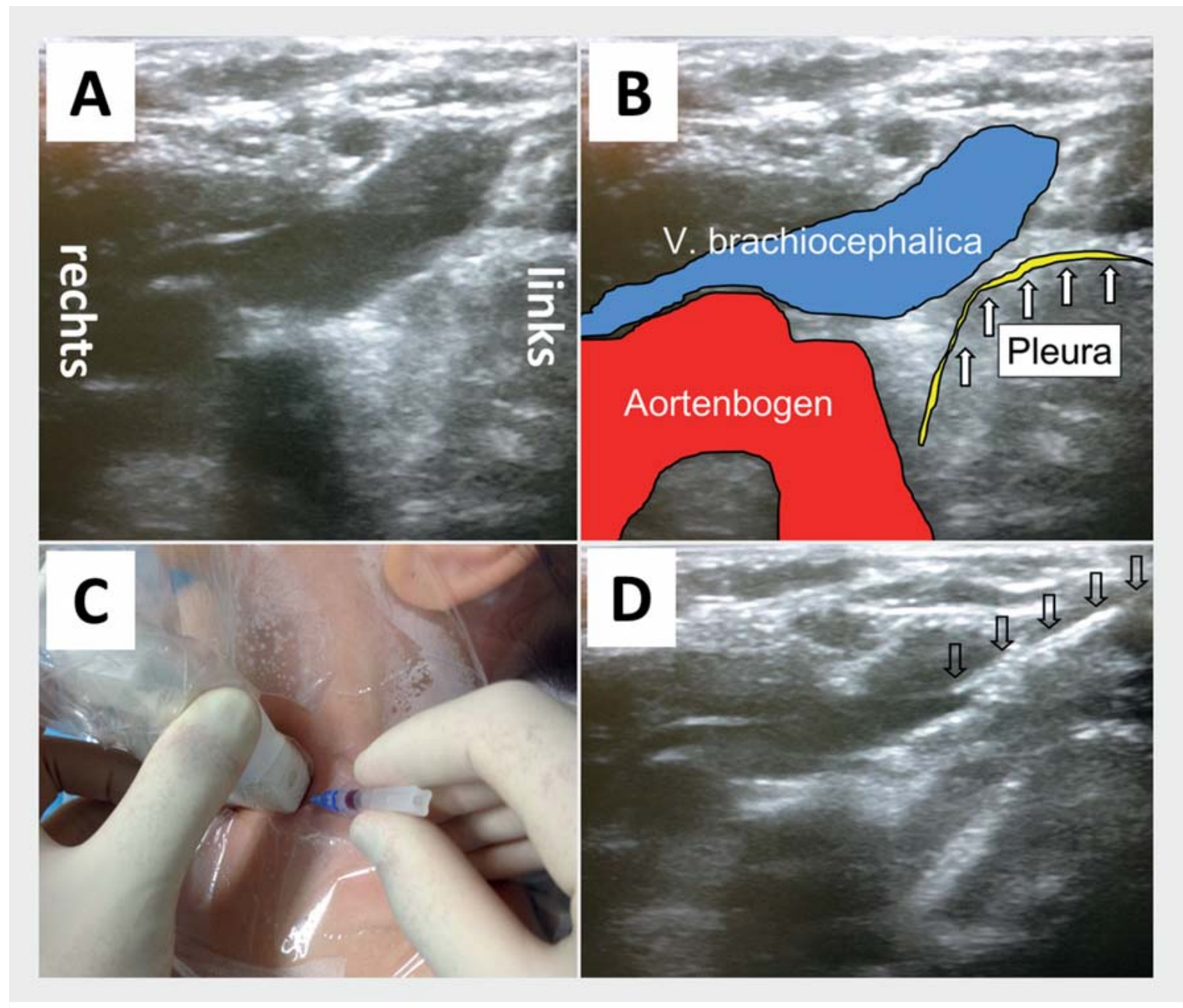

- Abb. 7 „Real-time-Ultrasound-guided-Technik“ des linkseitigen supraklavikulären Zugangs. (a, b) Sog. „lange Achse“-Blick (Long-Axis View, LAX) von V. brachiocephalica, Aortenbogen und Pleura. (c) Schallkopf in Position der langen Achse auf der Fossa supraclaviculare mit dargestellter Punktionskanüle (22 G). (d) Darstellung der Punktionskanüle in kompletter Länge. Die Spitze der Punktionskanüle hat die $\mathrm{V}$. brachiocephalica erreicht.

\section{Limitationen der Sonografie}

Nicht immer ist gewährleistet, dass besonders in Notfallsituationen passende, d.h. transportable Geräte schnell verfügbar sind. Der Vorteil der ultraschallgesteuerten Punktion ist nur dann gegeben, wenn die komplette Nadel (in-plane-Technik), oder die Nadelspitze (out-of-plane-Technik) kontinuierlich dargestellt wird. Ansonsten nimmt das Risiko von punktionsassoziierten Komplikationen analog zur traditionellen, an anatomischen Landmarken orientierten Technik zu.

Merke

Bei der ultraschallgestützten Punktion müssen die Punktionskanüle und das Zielgefäß kontinuierlich dargestellt werden. Hinreichende Übungen und Trainingsmöglichkeiten müssen jedoch geboten sein, um sich an die Ultraschalltechnik zu gewöhnen.

\section{Alternative Punktionsorte}

Ein weiterer interessanter Punktionsort ist nicht nur im Notfall die V. femoralis und wird für zentralvenöse Zugänge nicht selten ausgewählt. Die häufigsten Komplikationen hierbei sind die versehentliche Punktion der A. femoralis (ca. 10\%) und die Hämatombildung (ca. 5\%) [39, 40]. Thrombosen entstehen häufiger als an anderen Stellen. Als Grund dafür wird der relativ kleine Gefäßdurchmesser, verbunden mit einer Abflussbehinderung gesehen, da der zentralvenöse Katheter im Vergleich recht groß ist [41]. Zur Erleichterung der Punktion wird von manchen Autoren empfohlen, das Hüftgelenk auf der Punktionsseite leicht zu abduzieren [42]. Ein Problem bei Säuglingen und Neugeborenen sind die Windeln: Die Punktion der $\mathrm{V}$. femoralis findet hier in einem potenziell feuchte und möglicherweise kontaminierten Gebiet statt [41]. 
Traditionell wird besonders bei erwachsenen Patienten im Notfall gern die V. subclavia punktiert, da diese selten kollaptisch ist und auch mithilfe traditioneller Techniken schnell erreicht werden kann. Bei Kindern und Säuglingen erschwert häufig das ausgedehnte subkutane Fettgewebe die Punktion. Gefürchtet sind auch Komplikationen wie die fälschliche Punktion der A. subclavia oder Pleura mit nachfolgendem Pneumothorax [39-41, 43]. Ein Pneumothorax kann gerade bei instabilen Kindern oder Säuglingen rasch lebensbedrohlich sein.

\section{Supraklavikulärer Zugang als Alternative}

Eine gute Alternative dazu ist der linksseitige supraklavikuläre Zugang, der allerdings ausschließlich unter Zuhilfenahme des Ultraschalls mit direkter Visualisierung der Nadel vorgenommen werden sollte $[44,45]$. Bei dieser Methode wird ein zentralvenöser Katheter in die V. brachiocephalica gelegt. Der Punktionsort ist gut zugänglich und der Katheter ist später sicher und einfach zu fixieren. Wie - Abb. 7 zeigt, können die Pleura und der Aortenbogen mit Gefäßabgängen sowie das Zielgefäß, die V. bachiocephalica, in einem Schnitt dargestellt werden ( $\vee$ Abb. 7a und b). Die Punktionskanüle lässt sich in der sog. „langen Achse“ (Long-Axis View = LAX) in der kompletten Länge verfolgen ( $\bullet$ Abb. 7 c und d). Bei korrekter Visualisierung des Punktionsvorgangs ist die Verletzung der Pleura oder die artifizielle arterielle Punktion ausgeschlossen. Nach eigenen Erfahrungen, sollte der dafür infrage kommende zentralvenöse Katheter weniger als $1 / 3$ des Durchmessers des Zielgefäßes betragen, um einen ungehinderten venösen Blutfluss zu garantieren. In unserer Abteilung hat sich folgende Faustformel zur Abschätzung der Kathetergröße etabliert:

\section{ABSCHÄTZUNG KATHETERGRÖSSE}

Wähle den Durchmesser des zentralvenösen Katheters in French $(\mathrm{Fr})$ so, dass er kleiner als das Zielgefäß in $\mathrm{mm}$ ist $(1 \mathrm{~mm} \cong 3 \mathrm{Fr})$ :

zentralvenöser Katheter $(\mathrm{Fr})<$ Durchmesser Zielgefäß (mm)

Z. B. wenn das Zielgefäß im Ultraschall mit $4 \mathrm{~mm}$ bemessen wurde, sollte der zentralvenöse Katheter $<4$ Fr sein.

\section{Schlussfolgerungen}

Die rasche Etablierung eines Gefäßzugangs kann nicht nur beim pädiatrischen Patienten lebensrettend sein. Besonders aber in dieser Patientengruppe ist die Anlage eines Venenkatheters oft schwierig. Alternativ zu traditionellen Techniken, die sich an anatomischen Strukturen orientieren, setzt sich die Anwendung von Ultraschall als Standardverfahren immer mehr durch. Leichte, einfach zu transportierende und schnell bedienbare Geräte sind bereits kommerziell verfügbar. Auch die Technik des i. o. Zugangs muss erlernt und beherrscht werden, da diese schnell zu etablieren und auch bei Säuglingen und kleinen Kindern leicht anwendbar ist. Unabhängig davon sollte der Anwender die damit verbundenen Risiken und Komplikationen kennen.

\section{KERNAUSSAGEN}

- In Notfallsituationen wird die periphere Verweilkanüle oder der intraossäre Gefäßzugang empfohlen.

- Intraossär verabreichte Medikamente erreichen schnell den zentralen Kreislauf.

- Die aktuelle Leitlinie des European Resuscitation Council empfiehlt einen i. o. Zugang, sollte der i. v. Zugang nicht innerhalb von 2 min erfolgreich sein.

- Es wird empfohlen, den i. o. Zugang nach Stabilisierung der Vitalfunktionen und Transport ins Krankenhaus durch einen entsprechenden peripheren oder zentralen Venenkatheter zu ersetzen.

- Die Sonografie kann die Anlage von peripheren Verweilkanülen sowie zentralvenösen Kathetern beim pädiatrischen Patienten erheblich erleichtern und ist gerade in der Pädiatrie inzwischen klinischer Standard.

\section{Literatur}

[1] Maconochie IK, Bingham R, Eich C et al. European Resuscitation Council Guidelines for Resuscitation 2015: Section 6. Paediatric life support. Resuscitation 2015; 95: 223-248

[2] Rosetti VA, Thompson BM, Miller J et al. Intraosseous infusion: an alternative route of pediatric intravascular access. Ann Emerg Med 1985; 14: 885-888

[3] Seigler RS, Tecklenburg FW, Shealy R. Prehospital intraosseous infusion by emergency medical services personnel: a prospective study. Pediatrics 1989; 84: 173-177

[4] Ellemunter $\mathrm{H}$, Simma B, Trawoger $\mathrm{R}$ et al. Intraosseous lines in preterm and full term neonates. Arch Dis Child Fetal Neonatal Ed 1999; 80: F74-F75

[5] Fiorito BA, Mirza F, Doran TM et al. Intraosseous access in the setting of pediatric critical care transport. Pediatr Crit Care Med 2005; 6: 50-53

[6] Horton MA, Beamer C. Powered intraosseous insertion provides safe and effective vascular access for pediatric emergency patients. Pediatr Emerg Care 2008; 24: 347-350

[7] Hansen M, Meckler G, Spiro D et al. Intraosseous line use, complications, and outcomes among a population-based cohort of children presenting to California hospitals. Pediatr Emerg Care 2011; 27: 928-932

[8] Neuhaus D. Intraosseous infusion in elective and emergency pediatric anesthesia: when should we use it? Curr Opin Anaesthesiol 2014; 27: 282-287 
[9] Banerjee S, Singhi SC, Singh S et al. The intraosseous route is a suitable alternative to intravenous route for fluid resuscitation in severely dehydrated children. Indian Pediatr 1994; 31 : $1511-1520$

[10] Johnson L, Kissoon N, Fiallos M et al. Use of intraosseous blood to assess blood chemistries and hemoglobin during cardiopulmonary resuscitation with drug infusions. Crit Care Med 1999; 27: 1147-1152

[11] Berg RA. Emergency infusion of catecholamines into bone marrow. Am J Dis Child 1984; 138: 810-811

[12] Fiser DH. Intraosseous infusion. N Engl J Med 1990; 322: 1579-1581

[13] Andropoulos DB, Soifer SJ, Schreiber MD. Plasma epinephrine concentrations after intraosseous and central venous injection during cardiopulmonary resuscitation in the lamb. J Pediatr 1990; 116: 312-315

[14] Kanter RK, Zimmerman J], Strauss RH et al. Pediatric emergency intravenous access. Evaluation of a protocol. Am J Dis Child 1986; 140: 132-134

[15] Olaussen A, Williams B. Intraosseous access in the prehospital setting: literature review. Prehosp Disaster Med 2012; 27: 468-472

[16] Helm M, Haunstein B, Schlechtriemen T et al. EZ-IO(R) intraosseous device implementation in German Helicopter Emergency Medical Service. Resuscitation 2015; 88: 43-47

[17] Suominen PK, Nurmi E, Lauerma K. Intraosseous access in neonates and infants: risk of severe complications - a case report. Acta Anaesthesiol Scand 2015; 59: 1389-1393

[18] Hallas P, Brabrand M, Folkestad L. Complication with intraosseous access: scandinavian users' experience. West J Emerg Med 2013; 14: 440-443

[19] Hosokawa K, Kato H, Kishi $\mathrm{C}$ et al. Transillumination by lightemitting diode facilitates peripheral venous cannulations in infants and small children. Acta Anaesthesiol Scand 2010; 54: 957-961

[20] Katsogridakis YL, Seshadri R, Sullivan C et al. Veinlite transillumination in the pediatric emergency department: a therapeutic interventional trial. Pediatr Emerg Care 2008; 24: 83-88

[21] Johr M, Berger TM. Venous access in children: state of the art. Curr Opin Anaesthesiol 2015; 28: 314-320

[22] Samoya SW. Real-time ultrasound-guided peripheral vascular access in pediatric patients. Anesth Analg 2010; 111: 823-825

[23] Oakley E, Wong AM. Ultrasound-assisted peripheral vascular access in a paediatric ED. Emerg Med Australas 2010; 22: $166-170$

[24] Doniger S], Ishimine P, Fox JC et al. Randomized controlled trial of ultrasound-guided peripheral intravenous catheter placement versus traditional techniques in difficult-access pediatric patients. Pediatr Emerg Care 2009; 25: 154-159

[25] Triffterer L, Marhofer P, Willschke $\mathrm{H}$ et al. Ultrasound-guided cannulation of the great saphenous vein at the ankle in infants. $\mathrm{Br}$ J Anaesth 2012; 108: 290-294

[26] Lamperti M, Caldiroli D, Cortellazzi P et al. Safety and efficacy of ultrasound assistance during internal jugular vein cannulation in neurosurgical infants. Intensive Care Med 2008; 34 : 2100-2105

[27] Warkentine FH, Clyde Pierce M, Lorenz D et al. The anatomic relationship of femoral vein to femoral artery in euvolemic pediatric patients by ultrasonography: implications for pediatric femoral central venous access. Acad Emerg Med 2008; 15 : $426-430$
[28] Verghese ST, McGill WA, Patel RI et al. Ultrasound-guided internal jugular venous cannulation in infants: a prospective comparison with the traditional palpation method. Anesthesiology 1999; 91: 71-77

[29] Verghese ST, McGill WA, Patel RI et al. Comparison of three techniques for internal jugular vein cannulation in infants. Paediatr Anaesth 2000; 10: 505-511

[30] Grebenik CR, Boyce A, Sinclair ME et al. NICE guidelines for central venous catheterization in children. Is the evidence base sufficient? Br J Anaesth 2004; 92: 827-830

[31] Leyvi G, Taylor DG, Reith E et al. Utility of ultrasound-guided central venous cannulation in pediatric surgical patients: a clinical series. Paediatr Anaesth 2005; 15: 953-958

[32] Iwashima S, Ishikawa T, Ohzeki T. Ultrasound-guided versus landmark-guided femoral vein access in pediatric cardiac catheterization. Pediatr Cardiol 2008; 29: 339-342

[33] Schindler E, Schears G], Hall SR et al. Ultrasound for vascular access in pediatric patients. Paediatr Anaesth 2012; 22 : 1002-1007

[34] Hosokawa K, Shime N, Kato Y et al. A randomized trial of ultrasound image-based skin surface marking versus real-time ultrasound-guided internal jugular vein catheterization in infants. Anesthesiology 2007; 107: 720-724

[35] Turba UC, Uflacker R, Hannegan C et al. Anatomic relationship of the internal jugular vein and the common carotid artery applied to percutaneous transjugular procedures. Cardiovasc Intervent Radiol 2005; 28: 303-306

[36] Dolla D, Cavatorta F, Galli S et al. Anatomical variations of the internal jugular vein in non-uremic outpatients. J Vasc Access 2001; 2: 60-63

[37] Sulek CA, Gravenstein N, Blackshear RH et al. Head rotation during internal jugular vein cannulation and the risk of carotid artery puncture. Anesth Analg 1996; 82: 125-128

[38] Gwak MJ, Park JY, Suk EH et al. Effects of head rotation on the right internal jugular vein in infants and young children. Anaesthesia 2010; 65: 272-276

[39] McGee DC, Gould MK. Preventing complications of central venous catheterization. N Engl J Med 2003; 348: 1123-1133

[40] Graham AS, Ozment C, Tegtmeyer $\mathrm{K}$ et al. Videos in clinical medicine. Central venous catheterization. N Engl J Med 2007; 356: e21

[41] Casado-Flores J, Barja J, Martino R et al. Complications of central venous catheterization in critically ill children. Pediatr Crit Care Med 2001; 2: 57-62

[42] Suk EH, Lee KY, Kweon TD et al. Ultrasonographic evaluation of the femoral vein in anaesthetised infants and young children. Anaesthesia 2010; 65: 895-898

[43] Karapinar B, Cura A. Complications of central venous catheterization in critically ill children. Pediatr Int 2007; 49: 593-599

[44] Yamamoto T, Schindler E. Is the supraclavicular approach to the central vein still risky and taboo? Paediatr Anaesth 2015; 25: 1176-1178

[45] Breschan C, Platzer M, Jost R et al. Consecutive, prospective case series of a new method for ultrasound-guided supraclavicular approach to the brachiocephalic vein in children. $\mathrm{Br}$ J Anaesth 2011; 106: 732-737 
Über die Autoren

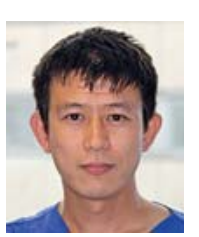

Tomohiro Yamamoto, MD, PhD

ist Facharzt für Anästhesie am Zentrum für Kinderanästhesiologie des Deutschen Kinderherzzentrums, Asklepios Klinik Sankt Augustin. E-Mail: t.yamamoto@asklepios.com

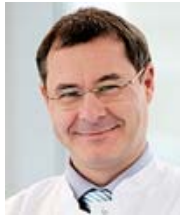

\section{Dr. med. Ehrenfried Schindler}

ist Chefarzt des Zentrums für Kinderanästhesiologie des Deutschen Kinderherzzentrums, Asklepios Klinik Sankt Augustin.

E-Mail: e.schindler@asklepios.com
Korrespondenzadresse

\section{Dr. med. Ehrenfried Schindler}

Asklepios Klinik Sankt Augustin GmbH

Deutsches Kinderherzzentrum (DKHZ)

Arnold-Janssen-Straße 29

53737 Sankt Augustin

e.schindler@asklepios.com

Interessenkonflikt

Die Autoren erklären, dass keine Interessenkonflikte vorliegen.

Bibliografie

DOI http://dx.doi.org/10.1055/s-0042-104853

Anästhesiol Intensivmed Notfallmed Schmerzther 2017; 52:

55-64 @ Georg Thieme Verlag KG Stuttgart · New York

ISSN 0939-2661 